\title{
Creating Culturally Safe Vocational Rehabilitation Services for Indigenous Australians: A Brief Review of the Literature
}

\author{
Vanette McLennan, ${ }^{1}$ Natalie Taylor, ${ }^{2}$ Amanda Rachow, ${ }^{2}$ Grant South ${ }^{2}$ \\ and Kelsey Chapman ${ }^{2}$ \\ ${ }^{1}$ School of Allied Health Sciences, Griffith University and Menzies Health Institute Queensland, \\ Gold Coast Campus, Parklands Drive, Southport, OLD, 4222, Australia \\ 2 School of Human Services and Social Work, Griffith University, Gold Coast Campus, Parklands \\ Drive, Southport, OLD, 4222 Australia
}

Whis article reviews the research evidence on vocational rehabilitation ser-
vices and rehabilitation counselling practice with Indigenous Australians.
It applied a context sensitive, cultural safety and reflexivity approach to con-
struct salient themes from the research evidence. Findings suggest Indigenous
Australians with disabilities experience significant 'double-disadvantage', and
that vocational rehabilitation service providers lack the knowledge and skills
in culturally safe practice in order to enhance the opportunities for social and
economic inclusion of Indigenous Australians. A whole-of-profession approach,
incorporating and ensuring Indigenous client equity and cultural safety in voca-
tional rehabilitation, is critically and urgently needed.

Keywords: vocational rehabilitation, Indigenous Australians, cultural safety

\section{Introduction}

The term 'Indigenous Australians' refers to a broad range of Aboriginal and Torres Strait Islander people considered as the first Australians with estimates suggesting a heritage of at least 50,000 years before the European settlement of Australia (Horton, 1994). It should be noted that although they are often grouped together as 'Indigenous Australians', Aboriginal and Torres Strait Islander people have different heritage and cultural histories, both between and within these groups. As of the 2011 Australian census, Indigenous Australians comprise 548,370 (approximately 4\%) of the country's total population of 21.5 million (Australian Bureau of Statistics (ABS), 2011a). They experience significant levels of social and economic disadvantage compared to fellow non-Indigenous citizens (Berry, Crowe, Deane, Billingaham, \& Bhagerutty,

Address for correspondence: Vanette E. J. McLennan, School of Allied Health Sciences, Griffith University and Menzies Health Institute Queensland, Gold Coast Campus, Parklands Drive, Southport, QLD, 4222. E-mail:v.mclennan@griffith.edu.au 
2012; Closing the Gap Clearinghouse (Australian Institute of Health and Welfare \& Australian Institute for Family Studies), 2013). For instance, 23.4\% of Indigenous people are living with a disability, 1.7 times the amount of non-Indigenous Australians (ABS, 2014a); Indigenous people are over-represented in the Australian prison population, where one in four of those imprisoned are Indigenous (SCRGSP (Steering Committee for the Review of Government Service Provision), 2014); and Indigenous household incomes are typically a fraction of non-Indigenous families (ABS, 2011b). High unemployment, more than three times higher than the unemployment rate of non-Indigenous working age Australians (ABS, 2014b), compounds the health and socioeconomic disadvantage for Indigenous people, their families and communities. These socioeconomic issues contribute to the multitude of barriers faced by Indigenous jobseekers, perpetuating the significant under-representation of Indigenous people in the workforce (National Centre for Vocational Education Research (NCVER), 2009), and even greater workforce exclusion experienced by Indigenous people with disability (ABS, 2014a).

Despite recognition of the positive impacts of workforce participation (The Royal Australasian College of Physicians and The Australasian Faculty of Occupational and Environmental Medicine, 2011), and the evidenced causal relationship between unemployment and psychological ill-health and social disadvantage (Stiglitz, 2002; Waters \& Moore, 2001; 2002), the vocational rehabilitation needs of Indigenous Australians living with injury and/or disability are relatively neglected compared to those of other segments of the population (Kendall \& Marshall, 2004). This might in part be from a lack of culturally competent vocational rehabilitation practice, which would go a long way to address work participation disadvantage amongst Indigenous people (Molloy \& Grootjans, 2014).

In response to these highlighted issues, the authors set out to perform a thematic review of literature pertaining to vocational rehabilitation of Indigenous people with disability, injury and/or chronic ill health. Upon searching, it became apparent that there has been next to no research or publication in this area, especially that relating specifically to vocational rehabilitation access and service provision for Indigenous Australians. First, the review considers the limited existing research as it relates to vocational rehabilitation of Indigenous people with disability or ill health, followed by a review of how the concept of cultural safety may be incorporated into vocational rehabilitation programmes. Finally, the review considers the implications for rehabilitation counselling and calls for more responsive vocational rehabilitation with Indigenous Australians.

\section{Method}

A search of scholarly journal articles published in the preceding 20 years (1995-2015 and later updated to include publications to mid-2016) was conducted, using the following search terms:

(aboriginal OR indigenous OR 'first nation' OR 'first people' OR 'first Australians') AND ('occupational rehabilitation' OR 'vocational rehabilitation' OR 'return to work' OR 'employment participation' OR 'workforce participation' OR 'employment services' 
OR 'career development' OR 'vocational counselling' OR 'vocational counselling' OR 'injury management' OR 'disability management').

Of the initial results, only eight articles in the Scopus database and nine generated in the Proquest database were remotely relevant to the topic based on the following exclusion criteria. The articles excluded from the review were those focussed on career development theory background, hospital/primary health care services and service provision outside of Australia. Initially, those excluded articles were categorised as such on the basis of not having a vocational rehabilitation or workforce participation focus, and/or not having relevance to the current context of vocational rehabilitation in Australia. However, the limited literature accessed using these exclusion criteria gave a very narrow view of the current state of vocational rehabilitation for ATSI people. Accordingly, the included literature was broadened to include 'cultural safety' and 'cultural humility', ensuring a more holistic picture of relevant historical, philosophical and practice-based considerations, not only regarding vocational rehabilitation with ATSI people but with Australian vocational rehabilitation more generally and discussions of cultural safety internationally.

\section{Findings}

The need to further examine cultural safety in regards to vocational rehabilitation practices with Indigenous Australians emerged as a critical focus given the scarcity of research in this field. There has been no specific research undertaken into culturally safe vocational rehabilitation in Australia, hence, a thematic review of the most relevant available literature was undertaken. Based on this review, the following areas are considered critical to starting a dialogue and taking 'first step' towards the construction of culturally safe vocational rehabilitation services with Indigenous Australians: the concept of 'cultural safety', knowledge of the meaning and experience of disability in Australian Indigenous communities and what culturally safe vocational rehabilitation practices may entail.

\section{Defining Cultural Safety and Related Concepts}

The concept of 'cultural safety' was first coined in health by Maori nurses in the 1990s (Papps \& Ramsden, 1996). To be culturally safe as a practitioner means to actively avoid any assault on, or risk to, a person's identity (Ramsden, 1990). It requires acceptance of cultural viewpoints and practices that are different from our own, whilst being mindful of broad individual and community differences across Indigenous Australia (Maher, 1999). Cultural safety is considered a more encompassing and inclusive approach to culture (Gerlach, 2012) than related notions of 'cultural competence' and 'cultural appropriateness', which focus on sensitivity in relation to ethnicity and cultural practices without challenging power or social inequities (DeSouza, 2008). The concept of cultural appropriateness calls for, perhaps unrealistically, the comprehensive cultural awareness training of health practitioners (Henderson, Kendall, \& See, 2011; McLennan \& Khavarpour, 2004; Westerman, 2004), and risks services being influenced by often essentialised and deterministic views, myths and stereotypes of Indigenous cultures, as well as the tendency to outsource 'cultural liaison' in disability and rehabilitation services (Hollinsworth, 2013). Instead, cultural safety is concerned 
with enabling health and social equity (Gerlach, 2012) by recognising the position of marginalised groups within an eco-systemic context of social, political and economic considerations, so that a more supportive practice of health service provision can be achieved (Smye \& Browne, 2002).

Relevant to cultural safety is the concept of cultural humility, first considered in health practice by Tervalon and Murray-Garcia (1998), which has since been linked to improved client-practitioner relationships in occupational therapy (Hammel, 2013), social work (Fisher-Borne, Cain, \& Martin, 2015) and nursing (Isaacson, 2014). A commitment to cultural humility in one's practice entails valuing life-long learning and critical self-reflection, along with a respectful and inquisitive approach whereby practitioners are expected to seek knowledge from their clients regarding their cultural and structural influences rather than assuming understanding or expertise about a culture outside of their own.

\section{Meanings and Experiences of Disability in Australian Indigenous Communities}

Beyond the more obvious aspects of disablement that result from functional impairments and social inequality for people with disability (Barnes \& Mercer, 2005), Indigenous people are confronted by additional layers of disablement from the effects of colonisation (Hollinsworth, 2013). There is now strong recognition that the trans-generational dispossession and social segregation of Indigenous peoples since colonisation, lie at the core of Indigenous disablement (Hollinsworth, 2013; King, Brough, \& Knox, 2014). This is compounded by the understandable suspicion and mistrust felt by some Indigenous Australians regarding the services offered to them, due to the untoward and disempowering impacts of previous government initiatives and services (NSW Ombudsman, 2010; Taylor, Thompson, \& Davis, 2009).

Indigenous community members may conceptualise disability and wellness in a vastly different way to traditional medical models of health (Australian Institute of Health and Welfare \& Australian Institute for Family Studies, 2013; Western Australia Disability Services Commission, 2009). For instance, some Indigenous Australians may understand health and wellness to be reflected in their inclusion and participation in their social and family groups rather than measured by objective or physical health markers, and, in another example, some Indigenous Australians perceive disability as something that should be accepted for 'what it is' rather than something that needs to be rehabilitated (King et al., 2014).

Indigenous Australians place a high value on inclusion and participation as well as connectedness with one's place and land, and the importance of family life and kinship ties (King et al., 2014). These values are in many ways at odds with the focus on individualism and self-determination (Morgan, Slade, \& Morgan, 1997) that are deeply embedded in dominant Western cultures and aligned services to Indigenous people with disabilities (Hasnain \& Sotnik, 2003). For instance, the common Indigenous preference for holistic and all-encompassing Aboriginal-controlled health services is not the model espoused in current disability and vocational rehabilitation service provision, in which a range of separate services are brokered and funded (Hollinsworth, 2013). Furthermore, specialist led models of care may serve to create abstract distinctions between person, place and society that conflict with Aboriginal philosophies of kinship and social interdependence (Morgan et al., 1997) and their preferences 
for integrated, holistic approaches to disability management and prevention (Kuipers, Harvey, Lindeman, \& Stothers, 2014). Further culturally nuanced differences between Indigenous and mainstream values in relation to disability management can be seen in the role of family and community leaders in decision-making. Whilst dominant service models may push for individual self-determination, Westby (2013) highlights how entire families, communities and elders are commonly involved in Indigenous decision-making around disability and its management.

Such culturally variant views of disability have profound implications for service design and usage. Indigenous populations may not see the value in services or interventions directed towards them (Westby, 2013), especially where policy and service designers have failed to articulate how disability support services may help clients improve their quality of life. Failure to recognise and respond to the specific needs and values of Indigenous people with disability will invariably lead to low service usage and in-turn the removal of disability funding when it is incorrectly perceived that disability services are no longer required (Kendall \& Marshall, 2004).

\section{Cultural Safety in Practice}

Research has found that Indigenous people value the inclusion of cultural considerations in treatment (Berry et al., 2012; Australian Institute of Health and Welfare \& Australian Institute for Family Studies, 2013). Whilst further investigation is required to establish whether cultural components enhance service goals and outcomes, it has been acknowledged that the stronger one's cultural identity, the greater the participation in education and training, and in-turn the likelihood of employment (NCVER, 2013). Despite inadequate research in this area, it is fair to propose the vocational rehabilitation practices that respect and support one's cultural identity and protocols will be far more client-centred and responsive than those that do not.

Unfortunately, there are no precedents for culturally safe vocational rehabilitation services in Australia, evidenced by the alarming lack of discussion or presentation of these in the literature. One pilot project, aiming to address vocational rehabilitation needs in the very remote Pitjantjatjara and Ngaanyatjarra communities in the adjoining corners of South Australia, Western Australia and The Northern Territory, was commenced by Arriotti (1995). The initiative was unfortunately discontinued due to reported resourcing issues. Over 10 years ago, Kendall and Marshall (2004) called for rehabilitation service providers to respond more appropriately to the vocational support needs of Indigenous Australia, however, this call remains unanswered to date.

Following consultations in Queensland with Indigenous health workers, community members and elders, Kendall, Milliken, Barnett and Marshall (2008) propose specific principles for culturally safe practice in Indigenous health, whereby influence is minimised to one's identity despite service delivery methods or processes being alien to the client's culture. These principles include acknowledgement of the need for cultural competence, respect for cultural protocols and Indigenous knowledge and the incorporation of these in practice where appropriate, and the ability to recognise concealed disability and appreciate relevant historical influences (Kendall et al., 2008). For health services to be more culturally safe, practitioners must engage in training, at the very least, in cultural awareness and culturally appropriate service provision (Henderson et al., 2011; McLennan \& Khavarpour, 2004; Westerman, 2004). It will 
require vocational rehabilitation practitioners to acknowledge their responsibility to understand and respect Indigenous concepts of health in order to meet the specific needs of Indigenous people (Kendall et al., 2008).

Further, the option of collaboration and inclusion of family members in all interventions and service planning should be part of a fundamental change to incorporate greater individual-directed control in Indigenous people's participation in rehabilitation (Arriotti, 1995; Kendall \& Marshall, 2004; Watts \& Carlson, 2002). A greater flow of knowledge and information between communities, workers, individuals and families will help to increase the cultural safety of all professional services provided to Indigenous communities (Kuipers et al., 2014). Rehabilitation professionals must engage in consultative relationships with clients and their families in order to identify unique local processes as well as the specific needs of that individual within their community.

\section{Discussion and Implications for Rehabilitation Counselling}

Rehabilitation counsellors are in a unique position to engage in a culturally safe manner with their clients due to their required training in socio-cultural issues related to health and disability. However, a recent review of contemporary vocational rehabilitation in Australia revealed that there is no explicit requirement for culturally appropriate communication with clients, beyond an expectation to demonstrate knowledge of 'psychosocial theory' (Buys, Matthews, \& Randall, 2014, p. 823), despite the diversity of individuals consulting rehabilitation counsellors in Australia. Kuipers et al. (2014) highlight the need for Indigenous health workers to demonstrate an awareness of chronic disease self-management, collaborative approaches to care and the ability to identify a client's strengths, capacities and psychosocial competence, all of which are well within the realm of rehabilitation counselling competencies (Matthews et al., 2015).

It is evident that rehabilitation counsellors have an opportunity to develop a deeper understanding of the unique cultural and socio-economic determinants of disability amongst Indigenous Australians, and further, how to address these issues with humility and commitment to cultural safety. Recent reviews of rehabilitation counselling competencies in Australia make note of the centrality of vocational rehabilitation to disability management (Matthews et al., 2015) and the recent shift towards people with disabilities seen as an untapped labour force (Buys et al., 2014). Despite this, there is no mention in the rehabilitation counselling literature of workforce participation for Indigenous people with disabilities or indeed the need for cultural safety when working with Indigenous populations.

An opportunity exists for rehabilitation counsellors to engage their communication skills to facilitate meaningful intercultural exchange and education between clients, communities, health professionals and potential employers. Maru and Davies (2011) suggest that whilst Indigenous people often have strong social 'bonding' networks, they commonly have narrow 'bridging' networks, which may limit access to social networks that support employment and serve as a disincentive to work. Kuipers et al. (2014) make a similar call for cross-cultural brokers to promote community engagement in health issues and build linkages, importantly, with a 
'two-way' approach that involves a flow of knowledge and information between workers, communities and individuals. Whilst supporting existing brokers is important (Maru \& Davies, 2011), there is also a need for new, culturally safe professionals to engage in intercultural brokerage.

For the rehabilitation counselling profession, this will require commitment to cultural safety as a core competency, involving relevant curriculum development in rehabilitation counselling education programs and dedicated continuing professional development in cultural safety for qualified practitioners. Such curricula and professional development must be constructed in consultation with Australian Indigenous service users, their families and communities, and could incorporate the use of the recently developed 'Cultural Responsiveness in Action: An IAHA Framework' (Indigenous Allied Health Australia, 2015) as an important foundational document. We propose that rehabilitation counsellors who feel competent in applying cultural safety across their scope of practice, and in engaging with the knowledge, values and understandings of the clients they serve, will be better placed to respond appropriately and more effectively to the unique needs of the individuals and communities with which they work. With the value placed on culturally relevant services by Indigenous people, assisting rehabilitation counsellors to develop culturally safe practice skills could help drive vocational rehabilitation service uptake, engagement and successful outcomes of Indigenous clients.

\section{Summary and Recommendations}

Cultural safety training is necessary in order to enable a greater appreciation for cultural traditions and knowledge, and this training must go beyond the tokenisation that has previously plagued professional preparation interventions (Hollinsworth, 2013; Kendall \& Marshall, 2004). It is essential that any culturally specific training is provided within a framework of person-centred practice to ensure that professionals enter the field with sufficient understanding and respect for cultural traditions, but with a commitment to openness to learning from and responding to their clients' unique experiences and needs. Furthermore, vocational rehabilitation professionals must continue to develop professional reflexivity in their work with Indigenous clients, their families and communities. This is perhaps best augmented by adopting the ethos of cultural humility (Hammel, 2013; Tervalon \& Murray-Garcia, 1998) as a guiding framework of culturally safe practice to ensure openness and accountability to clients' needs and their potential for meaningful community and workforce participation. Further, vocational rehabilitation approaches must include greater emphasis on community development and participation (Kuipers, Kendall, \& Hancock, 2001), and recognition of the potential for Indigenous community capacity building through skilled cultural brokerage and exchange (Kuipers et al., 2014).

For improved cultural safety in vocational rehabilitation services for Indigenous Australians, additions are needed to the existing rehabilitation counsellor education programs and professional development opportunities, so that cultural safety becomes a core competency of the rehabilitation counselling profession. In order for this to occur, the rehabilitation counselling profession itself must acknowledge the importance of cultural safety in practice, through actions such as updating 
accreditation requirements for rehabilitation counselling education programs, to ensure cultural safety is a key inclusion in university curricula. This will also require an active research agenda in this field. To date, there has been insufficient exploration of vocational rehabilitation policies and programs in the context of Indigenous communities. After completing the review of literature for this article, it is clear that further research, guided by Indigenous communities and rehabilitation service users, is urgently needed in order to develop a more complete picture of vocational rehabilitation service provision with Indigenous Australians.

Ensuring that vocational rehabilitation services are culturally safe and truly beneficial for Indigenous people with injury and disability is crucial to the effectiveness and integrity of the profession moving forward. The inclusion of culturally safe practice frameworks and application in work-integrated-learning contexts for rehabilitation counselling students is a necessary step towards ensuring the professional competencies of rehabilitation counsellors as culturally safe allied health practitioners.

\section{Financial Support}

We wish to acknowledge the School of Human Services and Social Work, Griffith University as a financial supporter of the research scholarship programme that enabled the writing of this paper.

\section{Acknowledgements}

This literature review was completed as part of a research scholarship programme in the School of Human Services and Social Work, Griffith University. The team consisted of Master of Rehabilitation Counselling student, Natalie Taylor, Master of Rehabilitation Counselling student and descendant of the Kamilaroi people, Amanda Rachow, Master of Philosophy student, qualified social worker and descendant of the Dalla People, Grant South, research support officer, Kelsey Chapman and qualified rehabilitation counsellor and supervising academic, Vanette McLennan. All authors share a common interest in improved vocational rehabilitation service delivery for Indigenous Australians.

\section{References}

ABS (Australian Bureau of Statistics). (2009a). Population Characteristics, Aboriginal and Torres Strait Islander Australians 2009. Cat. No. 6287.0. Canberra: Australian Bureau of Statistics.

ABS (Australian Bureau of Statistics). (2009b). Survey of Disability, Ageing and Carers 2009. Cat. No. 4430.0. Canberra: Australian Bureau of Statistics.

ABS (Australian Bureau of Statistics. (2011a). Census of Population and Housing: Counts of Aboriginal and Torres Strait Islander Australians. Cat No. 2075.0. Canberra: Australian Bureau of Statistics.

ABS (Australian Bureau of Statistics). (2011b). Census of Population and Housing: Characteristics of Aboriginal and Torres Strait Islander Australians 2011. Cat. No 2076.0. Canberra: Australian Bureau of Statistics.

ABS (Australian Bureau of Statistics). (2014a). Aboriginal and Torres Strait Islander People with a Disability, 2012. Cat. No. 4433.0.55.005. Canberra: Australian Bureau of Statistics.

ABS (Australian Bureau of Statistics). (2014b). Australian Social Trends, 2014. Cat. No. 4102.0. Canberra: Australian Bureau of Statistics. 
Arriotti, L. (1995). A tertiary rehabilitation pilot project in remote aboriginal communities. Proceedings from the 3rd National Rural Health Conference, Mt Beauty, 3-5 February 1995.

Barnes, C., \& Mercer, G. (2005). The Social Model of Disability: Europe and the Majority World (p. 218). Leeds: Disability Press.

Berry, S.L., Crowe, T.P., Deane, F.P., Billingaham, M., \& Bhagerutty, Y. (2012). Growth and empowerment for Indigenous Australians in substance abuse treatment. International Journal of Mental Health, 10(1), 970-983.

Buys, N., Matthews, L.R., \& Randall, C. (2014). Contemporary vocational rehabilitation in Australia. Disability $\mathcal{F}$ Rehabilitation, 37(9), 820-824. doi: 10.3109/09638288.2014.942001.

Closing the Gap Clearinghouse (Australian Institute of Health and Welfare \& Australian Institute for Family Studies). (2013). Strategies and practices for promoting the social and emotional wellbeing of Aboriginal and Torres Strait Islander people. Resource sheet no. 19. Canberra: Australian Institute of Health and Welfare \& Melbourne: Australian Institute of Family Studies.

DeSouza, R. (2008). Wellness for all: The possibilities of cultural safety and cultural competence in New Zealand. Journal of Research in Nursing, 13, 125-135.

Fisher-Borne, M., Cain, J.M., \& Martin, S.L. (2015). From mastery to accountability: cultural humility as an alternative to cultural competence. Social Work Education, 34(2), 165-181. doi: 0.1080/02615479.2014.977244.

Gerlach, A.J. (2012). A critical reflection on the concept of cultural safety. Canadian Journal of Occupational Therapy, 79(3), 151-158.

Hammel, K.R.W. (2013). Occupation, wellbeing, and culture: Theory and cultural humility. The Canadian Journal of Occupational Therapy, 80(4), 224-234. doi: 0.1177/0008417413500465.

Hasnain, R., \& Sotnik, P. (2003). Person-centred planning: A gateway to improving vocational rehabilitation services for culturally diverse individuals with disabilities. Journal of Rehabilitation, 69(3), 10-17.

Henderson, S., Kendall, E., \& See, L. (2011). The effectiveness of culturally appropriate interventions to manage or prevent chronic disease in culturally and liguistically diverse communities: A systematic literature review. Health and Social Care in the Community, 19(3), 225-249.

Hollinsworth, D. (2013). Decolonising Indigenous disability in Australia. Disability $\mathcal{G}$ Society, 28(5), 601-615. doi:10.1080/09687599.2012.717879.

Horton, D.R. (1994). Unity and Diversity: The History and Culture of Aboriginal Australia (Feature Article). Year Book Australia. Cat No. 1301.0. Canberra: Australian Bureau of Statistics.

Indigenous Allied Health Australia (IAHA). (2015). Cultural responsiveness in action: an IAHA framework. Canberra, ACT: IAHA. Available at http://iaha.com.au/wp-content/uploads/2015/ 08/2015-IAHA-Cultural-Responsiveness-Framework-WEB.pdf.

Isaacson, M. (2014). Clarrifying concepts: Cultural humility or competency. Journal of Professional Nursing, 30(3), 251-258. doi: 10.1016/j.profnurs.2013.09.011.

Kendall, E., \& Marshall, C.A. (2004). Factors that prevent equitable access to rehabilitation for aboriginal Australians with disabilities: The need for culturally safe rehabilitation. Rehabilitation Psychology, 49(1), 5-13. doi: 10.1037/0090-5550.49.1.5.

Kendall, E., Milliken, J., Barnett, L., \& Marshall, C. (2008). Improving practice by respecting indigenous knowledge and ways of knowing. In S. Taylor, M. Foster, \& J. Fleming (Eds.), Health Care Practice in Australia: Policy, Context and Innovations (pp. 220-237). Melbourne: Oxford Uni Press.

King, J.A., Brough, M., \& Knox, M. (2014). Negotiating disability and colonisation: The lived experience of Indigenous Australians with a disability. Disability $\mathcal{E}$ Society, 29(5), 738-750. doi:10.1080/09687599.2013.864257. 
Kuipers, P., Harvey, D., Lindeman, M., \& Stothers, K. (2014). Aboriginal and torres strait islander health practitioners in rural areas: Credentialing, context and capacity building. Rural and Remote Health, 14, 2897.

Kuipers, P., Kendall, E., \& Hancock, T. (2001). Developing a rural community-based disability service: (1) Service framework and stategy. Australian Journal of Rural Health, 9, 22-28.

Maher, P. (1999). A review of traditional Aboriginal health beliefs. Australian Journal of Rural Health, 7, 229-236.

Maru, Y.T., \& Davies, J. (2011). Supporting cross-cultural brokers is essential for employment among Aboriginal people in remote Australia. Rangeland Journal, 33(4), 327-338. doi: 10.1071/RJ11022.

Matthews, L.R., Buys, N., Randall, C., Marfels, B., Niehaus, M., \& Bauer, J.F. (2015). A comparative study of the job tasks, functions, and knowledge domains of rehabilitation professionals providing vocational rehabilitation services in Australia and Germany. Rehabilitation Counseling Bulletin, 58(2), 80-90.

McLennan, V., \& Khavarpour, F. (2004). Culturally appropriate health promotion: Its meaning and application in aboriginal communities. Health Promotion Journal of Australia, 15(3), 237-239.

Molloy, L., \& Grootjans, J. (2014). The ideas of frantz fanon and culturally safe practices for aboriginal and torres strait islander people in Australia. Issues in Mental Health Nursing, 35(3), 207-211.

Morgan, D.L., Slade, M.D., \& Morgan, C.M. (1997). Aboriginal philosophy and its impact on health care outcomes. Australian and New Zealand Journal of Public Health, 21, 597-601.

National Centre for Vocational Education Research (NCVER). (2009). Brokering successful Aboriginal and Torres Strait Islander Employment Outcomes: Common Themes in Good-Practice Models. Adelaide, SA: NCVER, Aus. Govt.

National Centre for Vocational Education Research (NCVER). (2013). Cultural Dimensions of Indigenous Participation in Vocational Education and Training: New Perspectives. Adelaide, SA: Australian Government.

NSW Ombudsman. (2010). Improving Service Delivery to Aboriginal People with a Disability: A Review of the Implementation of ADHC's Aboriginal Policy Framework and Aboriginal Consultation Strategy. A special report to Parliament under section 31 of the Ombudsman Act 1974, Sept 2010. Available at http://www.ombo.nsw.gov.au/_data/assets/pdf_file/ 0011/3350/SR_ImprvServDeliAboriginalPeopDisability_Sept10.pdf.

Papps, E., \& Ramsden, I. (1996). Cultural safety in nursing: The New Zealand experience. International Journal of Quality in Health Care, 8(5), 491-497.

Ramsden, I. (1990). Cultural safety. New Zealand Nursing Journal, 83, 18-19.

SCRGSP (Steering Committee for the Review of Government Service Provision). (2014). Overcoming Indigenous Disadvantage: Key Indicators 2014. Canberra: Productivity Commission.

Smye, V., \& Browne, A.J. (2002). 'Cultural Safety' and the analysis of health policy affecting Aboriginal people. Nurse Researcher, 9(3), 42-56.

Stiglitz, J.E. (2002). Employment, social justice and societal well-being. International Labour Review, 141, 9-29. doi: 10.1111/j.1564-913X.2002.tb00229.x.

Taylor, K., Thompson, S., \& Davis, R. (2009). Delivering culturally appropriate residential rehabilitation for urban Indigneous Australians: A review of the challenges and opportunities. Australian and New Zealand Journal of Public Health, 31(S1), S36-S40.

Tervalon, M., \& Murray-Garcia, J. (1998). Cultural humility versus cultural competence: A critical distinction in defining physician training outcomes in multicultural education. Journal of Health Care for the Poor and Undeserved, 9(2), 117-125. doi: 10.1353/hpu.2010.0233.

The Royal Australasian College of Physicians and The Australasian Faculty of Occupational and Environmental Medicine. (2011). Realising the Health Benefits of Work, A Position Statement. Available from http://www.racp.edu.au/docs/default-source/default-document-library/ afoem-pos-aus-nz-con-health-benefits-work-pack.pdf?sfvrsn=0. 
Waters, L.E., \& Moore, K.A. (2001). Coping with economic deprivation during unemployment. Journal of Economic Psychology, 22, 461-482. doi: 10.1016/S0167-4870(01)00046-0.

Waters, L.E., \& Moore, K.A. (2002). Reducing latent deprivation during unemployment: the role of meaningful leisure activity. Journal of Occupational and Organizational Psychology 75, 15-32. doi: $10.1348 / 096317902167621$.

Watts, E., \& Carlson, G. (2002). Practical stategies for working with indigenous people living in Queensland, Australia. Occupational Therapy International, 9(4), 277-293. doi: 10.1002/oti.169.

Westby, C. (2013). Implementing recommendations of the world report on disability for indigenous populations. International Journal of Speech-Language Pathology, 15(1), 96-100.

Westerman, T. (2004). Engagement of Indigenous clients in mental health services: What role do cultural differences play? Australian E-Journal for the Advancement of Mental Health, 3(3), 1-7.

Western Australia Disability Services Commission. (2009). Aboriginal People with Disabilities: Getting Services Right. Perth: Disability Services Commission, Government of Western Australia. Available from www.nds.org.au/asset/view_document/979317873. 